\title{
Fuzzy Fractional Order Sliding Mode Control for Automatic Clutch of Vehicle AMT
}

\author{
Yingchun Long ${ }^{1,2}$ and Lifu $\mathrm{Li}^{1}$ \\ ${ }^{1}$ School of Mechanical and Automotive Engineering South China University of \\ Technology, Guangzhou 510640, China \\ ${ }^{2}$ School of Physics and Mechanical \& Electrical Engineering, Shaoguan \\ University, Shaoguan 512005, China \\ ${ }^{1}$ sgulyc@139.com, ${ }^{2}$ pmlfli@scut.edu.cn
}

\begin{abstract}
The automatic clutch driving system of vehicle automated manual transmission (AMT) is a typical nonlinear system. A fast and accurate position control is difficult to achieve because of the nonlinearity of the load changes caused by the clutch diaphragm spring, the cushion spring, and other nonlinear components and because of the uncertainty of the load changes caused by the cushion spring deformation in the clutch engagement process at varying temperatures on the friction surface. In this paper, based on the theory of fractional calculus and traditional sliding mode control, the method of fuzzy fractional order sliding mode control (FFOSMC) is proposed for clutch control. By selecting the fractional order sliding surface, the laws of control are designed; and self-tuning of the switch gain is realized with the fuzzy controller. Theoretical analysis and numerical simulation revealed that compared with the traditional fuzzy integer order sliding mode controller, FFOSMC achieves better dynamic performance in position control and better robustness against load disturbance and other uncertain factors.
\end{abstract}

Keywords: Fuzzy fractional order sliding mode control, AMT, Clutch

\section{Introduction}

Automated manual transmission (AMT), which adopts the automatic clutch and automatic shift system on manual transmission, has realized automatic clutching and shifting with electronic control units; AMT has been widely used because of its high efficiency and low cost, among other advantages [1-3]. However, the typical AMT adopts a dry clutch structure. Thus, the nonlinear deformation of its internal diaphragm springs and cushion springs as well as the uncertainty of cushion spring deformation caused by temperature changes on the friction surface in the clutch engagement process [4] cause nonlinearity and uncertainty of the load changes for the clutch driving system. In addition, separation and engagement, particularly the engagement control when the vehicle is started, become difficult, thereby restricting AMT performance [6], because the parameters of the driving motor varies with time and because the process is influenced by the driver's intention [5].

Automatic operation laws and appropriate controlling methods must be established to realize real-time tracking of the automatic operation laws with the clutch actuator [7]. Controlling strategies and laws of operation [8-11] have been explored in previous studies on automatic clutch requirements, including small jerk and minor friction loss.

Real-time tracking of automatic clutch laws is completed by the driving system to control the position of the clutch actuator, including the release bearing and the pressure plate $[12,13]$. In the control process, (1) the accuracy of the position control directly affects the size of the clutch transfer torque and significantly 
influences the performance of the vehicle start and gear shift; (2) the speed of the position response, i.e., the response time of the clutch driving system, directly affects the dynamic performance of vehicles. However, an accurate and rapid position control is difficult to achieve because of the nonlinear characteristics of the dry clutch, time varying parameters, and other factors [14]. Various control methods have been proposed to overcome the problems of nonlinear control; these methods include flatness-based control [15], model predictive control [16], sliding mode control (SMC) [17-19], adaptive nonlinear observer [20], feed forward-feedback control [21], and adaptive Neuron PID control [22].

Among the abovementioned methods, SMC can realize robust control and is characterized by fast response, strong robustness to the uncertainty of system parameters, low sensitivity to external disturbance, and easy realization. [23], suggesting that SMC satisfactorily meets the control requirements of the automatic clutch system with nonlinearity and uncertainty. However, the essential discontinuous switching characteristic of the conventional SMC causes high frequency chatter. Thus, the switching function $\operatorname{sgn}(\cdot)$ is often replaced with the saturation function $\operatorname{sat}(\cdot)$ to avoid or completely eliminate the chatter. However, this technique does not guarantee that the steady-state tracking error of the system would converge to zero, thereby causing steady-state error. To solve this problem, Chern [24] proposed the integral sliding mode control, which lowers the transient performance while eliminating the steady-state error [25].

Fractional calculus, which extends the conventional integer order calculus to any order, is characterized by infinite dimension, hereditary, and memorization [26]. In a number of studies, fractional calculus and SMC have been combined and applied to nonlinear system control, thus weakening the chatter while maintaining the robustness and response characteristics of the traditional sliding mode control [2729].

On the basis of the accuracy and rapidity requirements for the position control of the automatic clutch driving system, as well as the strong-nonlinearity of the system itself, fuzzy fractional order sliding mode control (FFOSMC) is proposed in this study. A fractional order sliding surface is designed with regard to the slow energy transfer of the fractional order sliding surface, and real-time self-tuning sliding mode switch gain of the fuzzy controller is designed with regard to the uncertainty of parameters and disturbance changes. The objective of such a design is to weaken the chatter caused by the sliding mode control while improving the accuracy of the automatic clutch position control and the robustness against load disturbance, thereby realizing better control performance than that of the traditional integer order sliding mode control.

The remainder of this paper is organized as follows. Section 2 introduces the structure of automatic clutch and establishes the dynamic model. Section 3 describes the designing of the fractional order sliding mode controller and the fuzzy controller with self-tuning sliding mode switch gain. Section 4 analyzes the advantages of FFOSMC over the traditional SMC in terms of chatter, stability, and robustness. Section 5 presents the numerical simulation and results analysis. Section 6 provides the conclusions.

\section{Mathematical Modeling of the Clutch Driving System}

\subsection{Structure of the Clutch Driving System and Transmission Relations}

The structure of the automatic clutch system in this study is shown in Figure 1. The system is composed of a clutch motor (DC motor), a transmission unit (gear 
reduction unit and lever mechanism) and a clutch (diaphragm spring, pressure plate, clutch plate, and flywheel).

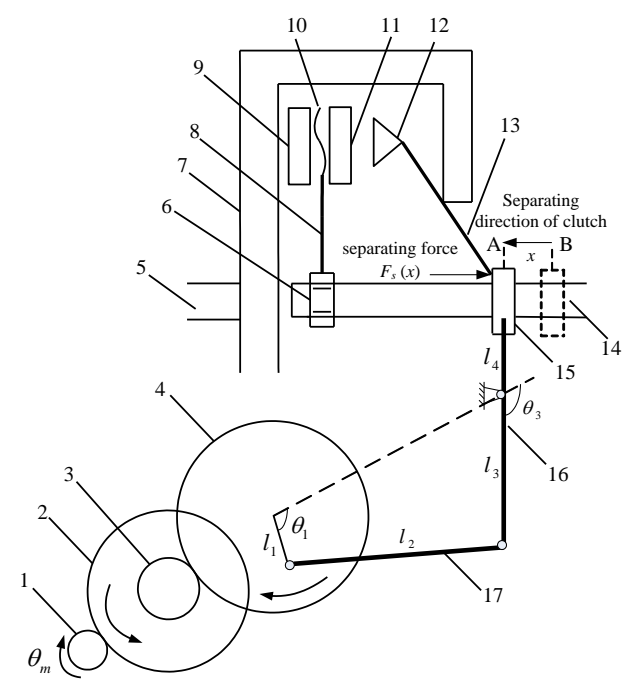

\section{Figure1. Schematic Diagram of the AMT Clutch System}

1. Output shaft gear of DC motor 2. First large reduction gear 3. Second small reduction gear 4. Second large reduction gear 5. Crankshaft 6. Drive plate hub 7. Flywheel 8. Clutch plate 9. Friction pad on the pressure plate side 10. Cushion spring 11. Friction pad on the flywheel side 12. Pressure plate 13. Diaphragm spring 14. Main shaft 15. Release bearing 16. Release lever 17. Push rod

As shown in Figure 1, the clutch motor rotating clockwise drives the gear retarding mechanism and the leverage mechanism drives the release bearing shifting from position $\mathrm{A}$ to position $\mathrm{B}$, thus realizing engagement through the diaphragm spring and the pressure plate. When the motor rotates counter-clockwise, clutch separation is realized.

The relationship between the displacement $x$ of the clutch release bearing and the rotation angle $\theta_{m}$ of the clutch motor in Figure 1 is expressed as

$$
x=f\left(\theta_{m}\right) \approx \frac{l_{4}}{i_{1} i_{2} i_{3}} \theta_{m}=k_{\theta} \theta_{m}
$$

where $k_{\theta}$ is constant; $i_{1}$ and $i_{2}$ are reduction ratios of the first and the second gear reduction mechanisms respectively; $i_{3}$ is the reduction ratio of the second reduction gear to the clutch fork determined by a lever mechanism composed of a push rod and a release lever:

$$
i_{3}=\frac{\dot{\theta}_{1}}{\dot{\theta}_{3}}
$$

Where $\theta_{1}$ and $\theta_{3}$ are the included angles of the lever mechanism in Figure 1, and their relations can be calculated with the following constraint kinematics equation:

$$
\theta_{3}=2 \operatorname{atan}\left(\frac{-B \pm \sqrt{B^{2}+A^{2}-C^{2}}}{A+C}\right)
$$


And $A=2 l_{3}\left(l_{4}-l_{1} \cos \theta_{1}\right), B=2 l_{1} l_{3} \sin \theta_{1}, C=l_{2}^{2}-l_{1}^{2}-l_{3}^{2}-l_{4}^{2}+2 l_{1} l_{2} \cos \theta_{1}, l_{1} \sim l_{4}$ are parameters of the lever mechanism shown in Figure 1.

\section{2 Relationship between the Separating Force of the Clutch and the Displacement of the Release Bearing}

The force exerted by the diaphragm spring on the release bearing in the moving process is translated into the load of the clutch drive motor with strong nonlinearity. This force is the separating force $F_{\mathrm{s}}(x)$ of the clutch. Displacement of the release bearing $x$ and the corresponding separating force $F_{\mathrm{S}}$ are measured in the experiment, the experimental data obtained are fitted by the six-time polynomial, and the $F_{\mathrm{S}}-x$ relation curve is obtained, as shown in Figure 2. When $x=0$, the release bearing is located at position $\mathrm{B}$, and the clutch engages completely; when $x=10 \mathrm{~mm}$, the release bearing is located at position $\mathrm{A}$, and the clutch separates completely.

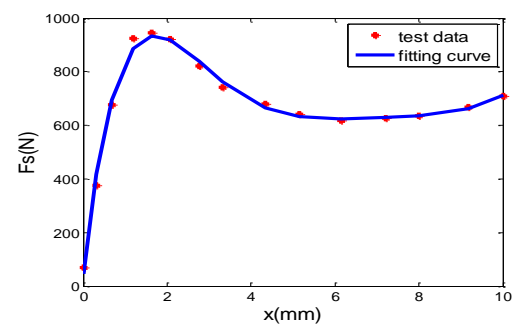

Figure2. Separation Characteristics Curve of the Clutch

The expression fitted by the six-time polynomial is as follows:

$F_{s}(x)=-0.03633 x^{6}+1.4718 x^{5}-23.5878 x^{4}+188.824 x^{3}-770.5284 x^{2}+1392.222 x^{1}+34.39$

(4)

\section{3 Dynamic Model of the Clutch Driving System}

A DC motor is used to drive the clutch, and the influence of the motor inductance is ignored. The voltage and torque equations of the motor can be expressed as follows:

(8)

$$
u=e+i_{a} R_{a}
$$

$$
T-T_{L}=J_{e q} \frac{d \omega_{m}}{d t}
$$

$$
T=C_{m} i_{a}
$$

$$
e=C_{m} \omega_{m}
$$

$$
\omega_{m}=\frac{d \theta_{m}}{d t}
$$

where $u$ is the motor voltage, $e$ is the motor induction electric potential, $i_{a}$ is the motor current, $C_{m}$ is the motor constant, $R_{a}$ is the total resistance of the armature circuit, $\omega_{m}$ is the motor angular rate, $J_{e q}$ is the equivalent to the moment 
of inertia of the motor rotor, $T_{L}$ is the equivalent load torque of the motor output shaft.

According to Equations (5)-(9),

$$
\frac{d^{2} \theta_{m}}{d t^{2}}=-\frac{C_{m}^{2}}{J_{e q} R_{a}} \frac{d \theta_{m}}{d t}+\frac{C_{m}}{J_{e q} R_{a}} u-\frac{1}{J_{e q}} T_{L}
$$

By substituting Equation (1) into Equation (10), the dynamic model of the clutch driving system is obtained as follows:

$$
\left\{\begin{array}{l}
\ddot{x}=-a \dot{x}+b u-c \\
a=\frac{C_{m}{ }^{2}}{J_{e q} R_{a}}, \quad b=k_{\theta} \frac{C_{m}}{J_{e q} R_{a}}, \quad c=k_{\theta} \frac{T_{L}}{J_{e q}}
\end{array}\right.
$$

Where

$$
J_{e q}=J+\frac{1}{i_{1}^{2}} J_{t 1}+\frac{1}{i_{1}^{2} i_{2}^{2}} J_{t 2}+\frac{1}{i_{1}^{2} i_{2}^{2} i_{3}^{2}} m_{b} l_{4}^{2}
$$

$$
T_{L}=i_{1} i_{2} i_{3}\left[F_{s}(x)-k_{m} \dot{x}\right] l_{4}
$$

where $J$ is the moment of inertia of the DC motor rotor and the output shaft gear, $J_{t 1}, J_{t 2}$ is the moment of inertia of the first reduction gear, $J_{t 2}$ is the equivalent moment of inertia of the second reduction gear and the lever mechanism, $m_{b}$ is the mass of the release bearing, and $k_{m}$ is the damping coefficient of the diaphragm spring.

In consideration of the influence of uncertainty and the time variant of system parameters and load disturbance, Equation (11) can be changed as follows:

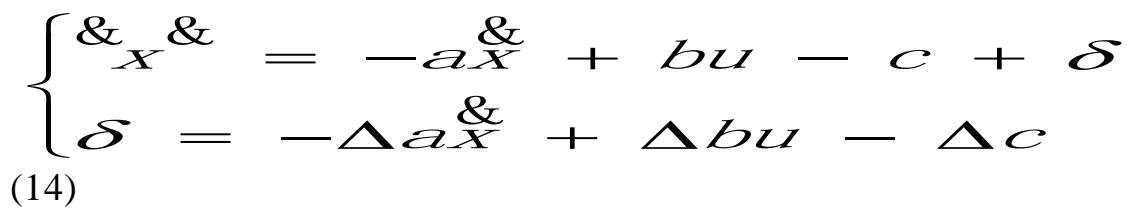

Where $\Delta a, \Delta b$ are system parameter perturbations, $\Delta c$ is the system load disturbance, and $\delta$ includes the function of time-varying parameters and load disturbance and is supposed to be limited, and

$$
|\delta| \leq \Psi, \quad \Psi \in \mathrm{R}^{+}
$$

\section{Design of the Fuzzy Fractional Order Sliding Mode Controller}

\subsection{Structure of the Control System}

The automatic clutch control system is a position servo mechanism used to track the target position of the clutch release bearing. The position of the release bearing is obtained by measuring the motor turn angle and conversion. Therefore, the structure of the control system is shown as follows: 


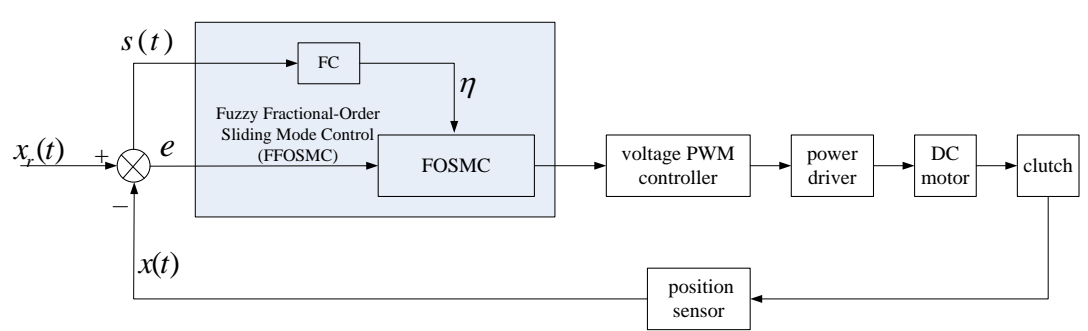

Figure 3. Structure of the Control System

In Figure 3, $x_{r}(t)$ is the target position value of the release bearing and is determined by TCU according to the driver's intentions, the working status of the engine, and ride Comfort [30]; $x(t)$ is the measured position value of the release bearing, i.e., the current actual position; and FFOSMC is composed of the fractional order sliding mode controller (FOSMC) and the fuzzy self-tuning module (FC) of the switch gain.

The controller generates appropriate clutch motor control signal, which drives the clutch motor and makes the release bearing position error tends to zero. Position deviation of the release bearing is defined as follows:

$$
e(t)=x_{r}(t)-x(t)
$$

According to Equation (16),

(17)

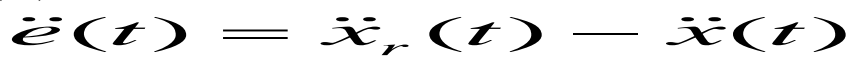

\subsection{Fractional Calculus}

Fractional calculus is a process of extending the classical integer order calculus to non-integral calculus. That is, the order can be arbitrary. The basic operation of the fractional calculus is defined as:

(19)

$$
{ }_{a} D_{t}^{r}= \begin{cases}\frac{d^{r}}{d t^{r}} & \operatorname{Re}(r)>0 \\ 1 & \operatorname{Re}(r)>0 \\ \int_{a}^{t}(d \tau)^{-r} & \operatorname{Re}(r)>0\end{cases}
$$

Where, $a$ and $t$ are the lower and upper limits of the basic operation, respectively; $r$ is the order of the fractional operation, and $\operatorname{Re}(r)$ is the real component of $r$.

In theoretical development, various definitions of fractional calculus are generated, and the definition of the commonly used Caputo fractional calculus [26] is shown as follows:

$$
{ }_{a} D_{t}^{r} f(t)=\frac{1}{\Gamma(m-r)} \int_{a}^{t} \frac{f^{m}(\tau)}{(t-\tau)^{a-m+1}} d \tau
$$

Where $m-1<r<m, \quad m \in N$.

Based on the definition of fractional calculus, fractional calculus has a higher degree of freedom than the integer calculus in order, and the fractional calculus of a 
continuous function at a certain point is related to the function values of all time points between the initial point and the specific point, and it has memory. Therefore, better control performance can be achieved by selecting the appropriate fractional order.

\subsection{Design of the Fractional Order Sliding Mode Controller}

The contents of design include sliding surface and control law.

Fractional order differential sliding surface is selected in this study.

$$
s(t)=k_{p} e(t)+{ }_{0} D_{t}^{r} e(t) \quad k_{p} \in \mathrm{R}^{+}, \quad r \in(0,1)
$$

Where $k_{p}$ is the gain of the sliding surface, and ${ }_{0} D_{t}^{r}(\cdot)$ is the fractional calculus operator.

Taking the derivative of Equation (21),

$$
\dot{s}(t)=\frac{d}{d t}\left(k_{p} e(t)+{ }_{0} D_{t}^{r} e(t)\right)=k_{p} \dot{e}(t)+{ }_{0} D_{t}^{r-1} \ddot{e}(t)
$$

Under ideal conditions ( $\delta=0$ ), when the system moves on the sliding mode, $\dot{s}(t)=0$, substitute Equations (14) and (18) into Equation (22):

$$
k_{p} \dot{e}(t)+{ }_{0} D_{t}^{r-1}\left(\ddot{x}_{r}(t)+a \dot{x}(t)-b u(t)+c(t)\right)=0
$$

Taking (1-r) order differential on both sides of Equation (23) with $D^{r}\left(D^{-r} f(t)\right)=f(t)$ [26], the equivalent control of the system in the sliding mode is obtained as follows:

$u_{e q}=b^{-1}\left(\ddot{x}_{r}(t)+a \dot{x}(t)+c(t)+k_{p 0} D_{t}^{1-r}(\dot{e}(t))\right.$

Considering the uncertainty of the parameters and load disturbance of the actual system, the following control law is adopted:

$$
\begin{aligned}
u & =u_{e q}+\eta \operatorname{sgn}(s) \\
& =b^{-1}\left(\ddot{x}_{r}(t)+a \dot{x}(t)+c(t)+k_{p 0} D_{t}^{1-r}(\dot{e}(t))\right)+\eta \operatorname{sgn}(s(t))
\end{aligned}
$$

where $\eta \operatorname{sgn}(s)$ is the switching control, realizing robust control of uncertainties of the system and external disturbance, $\eta$ is the switch gain, and $\operatorname{sgn}(\cdot)$ is the symbolic function and is defined as

$$
\operatorname{sgn}(s(t))=\left\{\begin{array}{cc}
1 & s(t)>0 \\
0 & s(t)=0 \\
1 & s(t)<0
\end{array}\right.
$$

Equation (25) is the control law and $r$ is the order of FOSMC. When the order $r$ is equal to 1, Equations (21) and (25) are the traditional integer order sliding surface and control law, respectively. That is,

$$
u=b^{-1}\left(\ddot{x}_{r}(t)+a \dot{x}(t)+c(t)+k_{p} \dot{e}(t)\right)+\eta \operatorname{sgn}(s(t))
$$




\subsection{Stability Analysis}

Stability analysis covers two aspects: (1) the control law determined by Equation (25) guarantees that the system can converge to the sliding mode at arbitrary initial state, thereby satisfying the reaching condition; and (2) when the system reaches the ideal sliding mode, the operation stability of the system on the fractional order sliding surface should be guaranteed.

Step 1: Reaching Condition

Theorem. For the clutch position control system of Equation (14), when the control law of Equation (25) is adopted, the sliding trajectory at arbitrary initial state will converge to the sliding surface determined by Equation (21).

Proof. Lyaponov function is selected as in reference [31]

$$
V=|s(t)|
$$

The derivative of $V$ is

$$
\dot{V}=\frac{d|s(t)|}{d t}=\operatorname{sgn}(s(t)) \cdot \dot{s}(t)
$$

According to Equations (14), (18) and (22), Equation (30) can be expressed as

$$
\dot{V}=\operatorname{sgn}(s(t)) \cdot\left(k_{p} \dot{e}(t)+{ }_{0} D_{t}^{r-1}\left(\ddot{x}_{r}(t)+a \dot{x}(t)-b u(t)+c(t)-\delta\right)\right)
$$

Substituting the control law of Equation (25) into Equation (31):

$$
\dot{V}=\operatorname{sgn}(s(t)) \cdot{ }_{0} D_{t}^{r-1}(-b \eta \operatorname{sgn}(s(t))-\delta)
$$

Then, the following two cases are discussed:

(a) When $s(t)<0, \dot{V}=-{ }_{0} D_{t}^{r-1}(b \eta-\delta)$; as $(r-1)<0$, when $\eta>\frac{\delta}{b}$, $\dot{V}<0$

(b) When $s(t)>0, \dot{V}=-{ }_{0} D_{t}^{r-1}(b \eta+\delta)$; as $(r-1)<0$, when $\eta>-\frac{\delta}{b}, \dot{V}<0$

According to Equation (15), when the switch gain satisfies

$$
\eta>\frac{|\psi|}{b}
$$

Then $\dot{V} \leq 0$. Based on Lyaponov stability theorem, the reaching condition is satisfied.

The proof has been completed.

Step 2: Stability

When the sliding mode occurs, Equation (21) can be rewritten as

$$
{ }_{0} D_{t}^{r} e(t)=-k_{p} e(t) \quad r \in(0,1)
$$

As $\left|\arg \left(-k_{p}\right)\right|=\pi>r \pi / 2$, according to stability theorem of the fractional order system [32], the sliding mode operation of the system is asymptotically stable.

\subsection{Fuzzy Self-tuning of Switch Gain}

Switch gain $\eta$ satisfies that Equation (33) is a condition of sliding mode convergence, but its size is a major cause of system chatter on the sliding surface. System disturbance $\delta$ varies with time. Thus, to reduce the chatter caused by $\eta, \eta$ 
should also be time-varying. However, tuning of the switch gain $\eta$ is very difficult because of the uncertainty of the system disturbance and the difficulty in measurement. Therefore, fuzzy self-tuning of switch gain is proposed in this paper.

Input/output fuzzy sets of the fuzzy controller are defined as follows:

$s=\eta=\{\mathrm{NZP}\}$, where the linguistic terms $\mathrm{N}, \mathrm{Z}$, and $\mathrm{P}$ respectively represent Negative, Zero and Positive, and their membership functions are shown in Figures 4 and 5 .

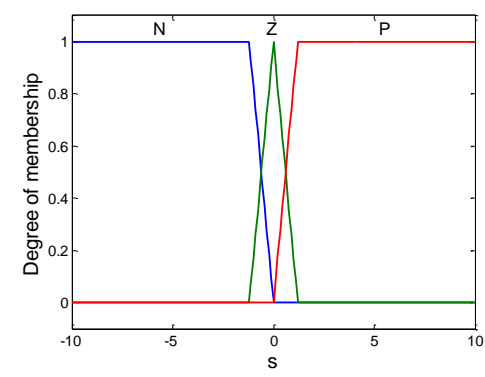

Figure 4. Membership Functions of $s$

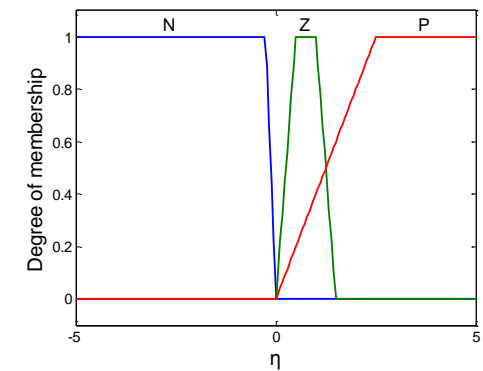

Figure5. Membership Functions of $\eta$

The fuzzy rules are designed as follows:

$\mathrm{R} 1$ : If $s$ is $\mathrm{N}$ then $\eta$ is $\mathrm{P}$;

$\mathrm{R} 2$ : If $s$ is $\mathrm{Z}$ then $\eta$ is $\mathrm{Z}$;

R3: If $s$ is $\mathrm{P}$ then $\eta$ is $\mathrm{N}$;

\section{Performance Analysis of the FOSMC}

The advantages of FOSMC over the traditional SMC are analyzed in terms of chatter restraining, stability, and robustness.

\subsection{Chatter}

Equation (34) is the fractional order linear time-invariant system, without loss of generality, and is expressed as follows:

$$
{ }_{0} D_{t}^{r} x(t)=A x(t) \quad A \in \mathrm{R}^{-}, \quad 0<r \leq 1
$$

The general solution of equation (35) is [27]:

$$
x(t)=\Phi(t) \cdot x(0)=E_{r, 1}\left(A t^{r}\right) \cdot x(0)
$$


Where $E_{r, 1}\left(A t^{r}\right)=\sum_{k=0}^{\infty} \frac{\left(A t^{r}\right)^{k}}{\Gamma(r k+1)}$ the state transition function of Equation (35) is' $\Gamma(z)$ is the Gamma function defined as $\Gamma(z)=\int_{0}^{\infty} t^{z-1} e^{-t} d t$.

When $\mathrm{r}=1$, Equation (35) is the integer order system $\dot{x}(t)=A x(t)$, and the state transfer function is

$$
E_{r, 1}\left(A t^{r}\right)=E_{1,1}(A t)=\sum_{k=0}^{\infty} \frac{(A t)^{k}}{\Gamma(k+1)}=\sum_{k=0}^{\infty} \frac{(A t)^{k}}{k !}=e^{A t}
$$

When $0<\mathrm{r}<1$, based on the results of Matignon [32],

$$
E_{r, 1}\left(A t^{r}\right)=\sum_{k=0}^{\infty} \frac{\left(A t^{r}\right)^{k}}{\Gamma(r k+1)} \sim \frac{1}{\Gamma(1-r)} A^{-1} t^{-r}
$$

Equations (37) and (38) show that the state $x(t)$ of integer order system towards 0 like $e^{A t}$ and fractional order system decays towards 0 like $t^{-r}$. It means that the energy transfer is slower with fractional order sliding surface than that with integer order counterpart [27]. Therefore, FOSMC adjusts the convergence of the sliding surface by adjusting $r$ to transfer energy slowly, which is conducive to reducing chattering amplitude.

In addition, the sliding surface of the fractional order determined by Equation (21) is adjusted as

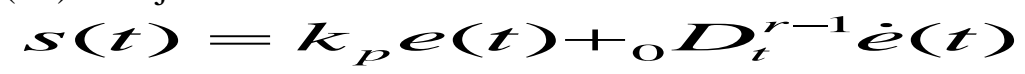

$0<\mathrm{r}<1$, indicating that sliding surface $s(t)$ determined by Equation (39) contains $|r-1|$ order integral of $\dot{\boldsymbol{e}}(\boldsymbol{t})$ and is thus smoother than the integer order sliding surface $s(t)$ determined by Equation (27). Therefore, the switching function of $\operatorname{sgn}(s)$ resulting from changing the sliding surface $s(t)$ itself is inhibited to a certain degree, and chatter caused by rapid changes of $u(t)$ in Equation (25) will be inhibited.

\subsection{Stability}

Ahmed [34] pointed out that systems with memory are typically more stable than their memory-less counterparts. Therefore, fractional order differential equations are, at least, as stable as their integer order counterparts. The clutch position servo system determined in Figure 3 adopts the FOSMC controller, which is a fractional order system. Consequently, the degree of achieving stability is increased. [35].

Furthermore, stability regions of the fractional order system $(0<r<1)$ that adopts sliding surface Equation (21) is shown in Figure 6(a), and it has a greater stability region than that of the integer order system $(r=1)$, as shown in Figure 6(b), suggesting that FOSMC has higher robustness. 


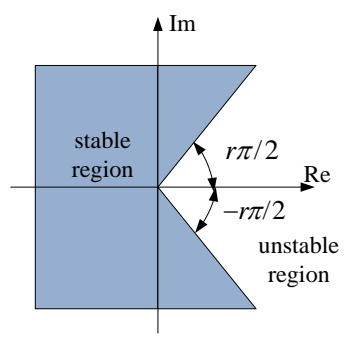

(a) $0<r<1$

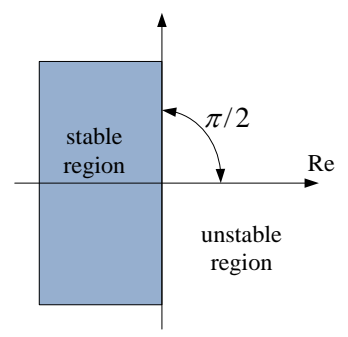

(b) $r=1$

Figure 6. Stability Regions of the Fractional Order System [36]

\section{3 Robustness}

By comparing the Lyaponov function differential expressions of FOSMC and SMC control methods, Equation (32) can be rewritten as

$$
\dot{V}_{F O S M C}=\operatorname{sgn}(s(t)) \cdot{ }_{0} D_{t}^{r-1}(-b \eta \operatorname{sgn}(s(t))-\delta)
$$

Similar to analysis process of Equations (29)-(32), the Lyaponov function differential expression of the traditional SMC can be obtained as

$$
\dot{V}_{S M C}=\operatorname{sgn}(s(t)) \cdot(-b \eta \operatorname{sgn}(s(t))-\delta)
$$

Then, the following two cases are discussed.

(a) When the system reaches the sliding mode, and $s(t)<0, \dot{V}<0$

If there is an instant disturbance $\delta_{\text {ins }}>|\psi|$ (such as mutation of load torque), $\dot{V}_{S M C}>0$ in Equation (41), the traditional SMC system would immediately lose stability. By contrast, for the FOSMC system, according to Equation (40), despite a $\delta_{\text {ins }}$ mutation disturbance $\left(\delta_{\text {ins }}>|\psi|\right)$, as $r-1<0$, the integral effect of the fractional order integral operation ${ }_{0} D_{t}^{r-1}(\cdot)$ prevents $\dot{V}_{F O S M C}$ from mutating, and $\dot{V}_{F O S M C}$ remains $\dot{V}_{F O S M C}<0$ in a short period of time. That is, the system will remain stable for an extra short period of time.

(b) When the system reaches the sliding mode, and $s(t)>0, \dot{V}<0$

If there is an instant disturbance $\delta_{i n s}<-|\psi|$ (such as mutation of load torque), as in the previous analysis, the traditional SMC system will immediately lose stability, whereas the FOSMC system, because of the integral effect of fractional order integral operation ${ }_{0} D_{t}^{r-1}(\cdot)$, will remain stable for an additional short period of time.

These analyses show that FOSMC shows stronger robustness than the traditional SMC method.

\section{Numerical Simulation and Result Analysis}

On the basis of Figures 1 and 3, the clutch simulation model is established in MATLAB/Simulink, and the main simulation parameters are listed in Table 1. 
Table 1. The Clutch System Parameters

\begin{tabular}{cl|ll}
\hline$R_{a}$ & $0.347(\Omega)$ & $l_{1}$ & $30 \mathrm{~mm}$ \\
$L$ & $6 \times 10^{-4}(\mathrm{H})$ & $l_{2}$ & $173.4 \mathrm{~mm}$ \\
$c_{m}$ & 0.026 & $l_{3}$ & $145.36 \mathrm{~mm}$ \\
$i_{1}$ & $59 / 9$ & $l_{4}$ & $87.82 \mathrm{~mm}$ \\
$i_{2}$ & $63 / 11$ & $J_{\text {eq }}$ & $4.2 \times 10^{-4}\left(\mathrm{Nms}^{2}\right)$ \\
\hline
\end{tabular}

To present a contrast, the traditional fuzzy integral sliding mode control (FISMC) is also simulated. $k_{p}=10$ And $r=0.8$ are adopted for the FFOSMC controller, and $k_{p}=10 r=1$ for the FISMC controller.

\section{1 Dynamic Characteristics Comparison under the Function of Step Signals}

Unit step signals are detected in the reference position of the release bearing, and a constant load torque $T_{L}=0.2 \mathrm{Nm}$ is exerted. The position tracking curves and the sliding surface convergence trajectories under FFOSMC and FISMC are shown in Figures 7 and 8, respectively.

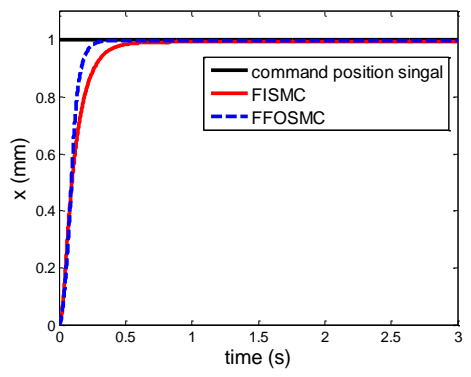

\section{Figure7. Position Unit Step Signal Response}

As shown in Figure 7, the rise time of FFOSMC is $0.25 \mathrm{~s}$, and that of FISMC is $0.5 \mathrm{~s}$, suggesting that FFOSMC control can respond faster.

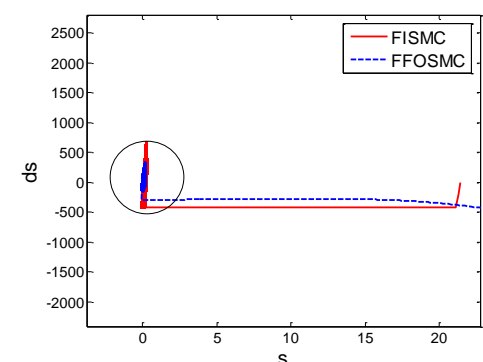

(a)

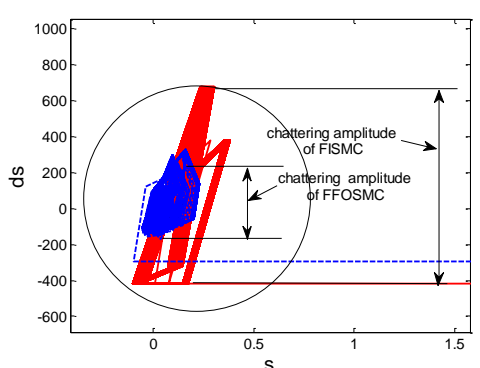

(b)

Figure8. Convergence Trajectory of the Sliding Surface

In Figure $8, d$ s of the ordinate are derivative of the sliding surface. That is, the trajectory change rate, $\mathrm{s}$, of the abscissa is the trajectory of the sliding surface.

Figure $8(\mathrm{~b})$ is the enlarged view of Figure 8(a) that shows the sliding surface convergence trajectory at the convergence point. As shown in the figure, FFOSMC and FISMC have chatter approximating the equilibrium state, but FFOSMC chatter amplitude is far less than that of FISMC. Therefore, FFOSMC reaches the steady state more smoothly and has an obvious dynamic quality that is superior to FISMC. 


\section{2 Stability Comparison under the Load Disturbance}

$\eta_{\text {mac }}=5$ Output amplitude of switch gain fuzzy controller is adopted. According to Equation (33) and the parameters given in Table 1, the maximum load disturbance of the system is $T_{\text {int(max) }}=0.378 \mathrm{Nm}$. Unit step signals are detected in the reference position of the release bearing. When the load disturbance signal shown in Figure 9 is applied to the system, the position tracking curves of the two control methods are shown in Figure 10.

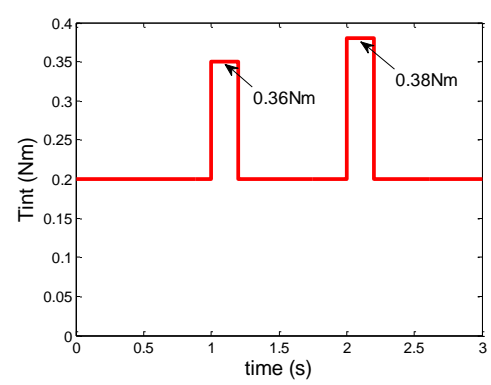

Figure 9. Load Disturbance Torque Curve

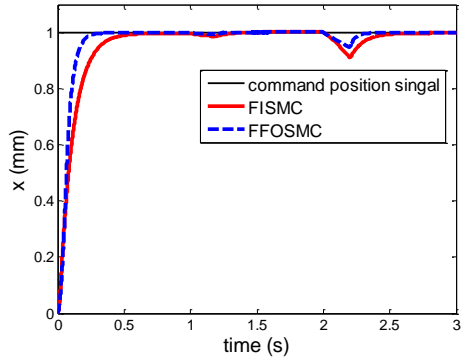

Figure10. Position Tracking Curve under Load Disturbance

According to Figure 10, in the interval between 1.1 and $1.2 \mathrm{~s}$, the load disturbance is below $T_{\text {int(max) }}$, and the system remains stable when the two methods of control is adopted. At $2 \mathrm{~s}$, under $0.38 \mathrm{Nm}$ impact load, greater than $T_{\text {int(max) }}$, the system becomes instable. However, the position deviation of FFOSMC is obviously less than that of FISMC, indicating that FFOSMC has stronger robustness than FISMC, which is consistent with the results of Section 4.3.

\subsection{Comparison of the Effect of time Delays on System Steady-state Error}

Ramp input signals are detected in the reference position of the release bearing, with a rise time of $0.5 \mathrm{~s}$, a steady state value of $1 \mathrm{~mm}$, and a load torque of $0.2 \mathrm{Nm}$. Figure 11(a) is the position tracking steady-state error curve without considering the influence of feedback delay. Figure 11(b) is the position tracking steady-state error curve when the position feedback delay influence caused by time and space hysteresis of the actual system is considered. In the simulation model, the position feedback delay time is set to $0.002 \mathrm{~s}$. 


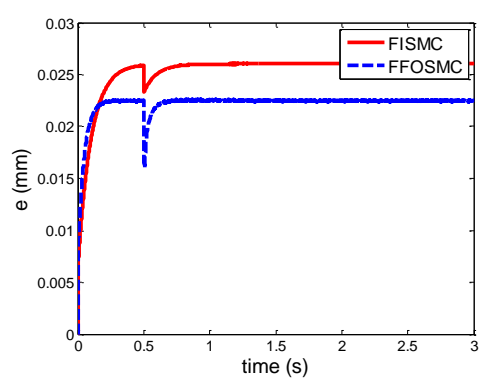

(a)

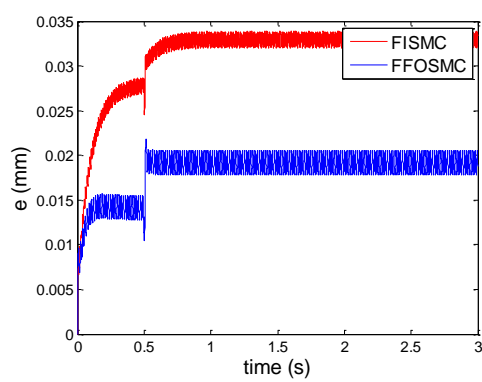

(b)

Figure 11. Position Tracking Steady-state Error Curve

According to Figure 11, in the absence of position feedback delay, the position tracking steady-state error of FFOSMC is approximately $0.022 \mathrm{~mm}$, and that of FISMC is approximately $0.026 \mathrm{~mm}$. When the position feedback delay is $0.002 \mathrm{~s}$, the position tracking steady-state error of FFOSMC is approximately $0.02 \mathrm{~mm}$, and that of FISMC is approximately $0.033 \mathrm{~mm}$. The curve shows that FFOSMC produces lower position steady-state error when there is position feedback delay.

\section{4 Comparison of the Clutch Position Trackings at Vehicle Starting}

According to the method in the literature [30], the target displacement curve of the release bearing is obtained under the condition of the vehicle starting with constant engine rotating speed, as shown in Figure 12. The period before $0.8 \mathrm{~s}$ is a process of clutch separation, and complete separation is realized. After $0.8 \mathrm{~s}$, clutch engagement is realized (i.e., vehicle is started).

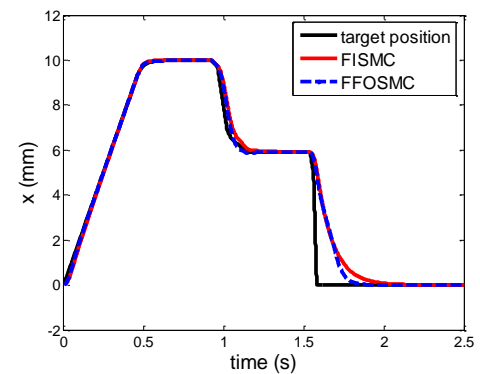

Figure 12. Clutch Position Tracking Curve at Starting

Simulation results reveal that both control methods have realized target position tracking, but FFOSMC achieves better dynamic performance, and it has a better and faster tracking ability when the target position changes rapidly.

\section{Conclusions}

A nonlinear robust controller based on fuzzy fractional order sliding mode control is proposed in this paper. The stability of this method is proved by Lyapunov theorem, and its robustness is analyzed. The self-tuning of switch gain of sliding mode controller is realized. Numerical simulation shows that for reasonable selection of the order $r$ of fractional order sliding surface, the proposed method is more accurate in position tracking, more efficient in dynamic performance, and stronger in robustness than the traditional FISMC. 


\section{References}

[1] G. Lucente, M. Montanari and C. Rossi, "Modelling of an automated manual transmission system", Mechatronics, vol. 172, (2007), pp. 73-91.

[2] L. Ling, Z. S. Chen and Y. Y. Luo, "Study on Control Process of Automatic Mechanical Transmission System for Pure Electric Bus", Applied Mechanics and Materials, vol. 220, (2012), pp. 1795-1799.

[3] M. G. Montazeri and Z. Pourbafarani, "Simultaneous design of the gear ratio and gearshift strategy for a parallel hybrid electric vehicle equipped with AMT", International Journal of Vehicle Design, vol. 582, no. 291-306, (2012).

[4] V. D'Agostino, N. Cappetti, M. Pisaturo and A. Senatore, "Dry clutch models for control of AMTs/DCTs: influence of the temperature level on frictional and elastic characteristics", International Journal of Mechanical Engineering and Industrial Design, vol. 1, no. 8, (2012), pp. 106-116.

[5] B. Z. Gao, H. Chen and X. H. Lu, "Improved optimal controller for start-up of amt trucks in consideration of driver's intention”, International Journal of Automotive Technology, vol. 14, no. 2, (2013), pp. 213-220.

[6] H. Y. Jin, X. S. Cheng and Y. D. Song, "Optimal Fuzzy Control of Automated Mechanical Transmission Clutch Engagement during Start-Up Process”, Advanced Materials Research, vol. 694-697, (2013), pp. 2089-2093.

[7] F. Liu, Y. X. Li, J. W. Zhang and H. C. Huang, "Robust control for automated clutch of AMT vehicle", SAE Paper, (2002).

[8] H. Tanaka and H. Wada, "Fuzzy control of engagement for automated manual transmission", Vehicle System Dynamics, vol. 24, (1995), pp. 365-366.

[9] B. Gao, X. Lu, H. Chen, "Dynamics and control of gear upshift in automated manual transmissions", International Journal of Vehicle Design, vol. 63, no. 1, (2013), pp. 61-83.

[10] T. Jin, P. Li and G. Zhu, "Optimal decoupled control for dry clutch engagement", American Control Conference ACC, IEEE, (2013).

[11] P. J. Dolcini, C. C. de Wit and H. Bechart, "Observer-based optimal control of dry clutch engagement", Oil \& Gas Science and Technology, vol. 62, no. 4, (2007), pp. 615-621.

[12] J. Kim, S. B. Choi, "Design and modeling of a clutch actuator system with self-energizing mechanism", Mechatronics, IEEE/ASME Transactions, vol. 16, no. 5, (2011), pp. 953-966.

[13] M. Montanari, F. Ronchi and C. Rossi, "Control and performance evaluation of a clutch servo system with hydraulic actuation", Control Engineering Practice, vol. 12, (2004), pp. 1369-1379.

[14] J. Horn, J. Bamberger and P. Michau, "Flatness-based clutch control for automated manual transmissions", Control Engineering Practice, vol. 12, (2003), pp. 1353-1359.

[15] R. Gasper, M. G. Chavez and D. Abel, "Adaptive Flatness Based Control of a Hydraulic Clutch Actuator", 8th IFAC Symposium on Nonlinear Control Systems, (2010).

[16] D. Schindele, R. Prabel and H. Aschemann, "Nonlinear Model Predictive Control of an Electropneumatic Clutch for Truck Applications", Preprints of the 7th Vienna Conference on Mathematical Modelling, (2012); Mathmod, Vienna.

[17] R. Prabel, D. Schindele and H. Aschemann, "Model-based control of an electro-pneumatic clutch using a sliding mode approach", Industrial Electronics and Applications ICIEA, 7th IEEE Conference, IEEE, (2012) .

[18] R. Knoblich, C. Guhmann and J. Beilharz, "Sliding mode position control for automotive dry clutch system: Nonlinear observer/sliding mode control design for an electro-hydraulic actuation system", 9th International Multi-Conference on Systems, Signals and Devices, IEEE, (2012).

[19] Y. S. Zhao, L. P. Chen and Y. Q. Zhang, "Enhanced fuzzy sliding mode controller for automated clutch of AMT vehicle", SAE Technical Paper, (2006).

[20] H. Langjord, G. O. Kaasa and T. A. Johansen, "Adaptive nonlinear observer for electropneumatic clutch actuator with position sensor", Control Systems Technology, IEEE Transactions, vol. 204, (2012), pp. 1033-1040.

[21] J. Deur, V. Ivanović and Z. Herold, "Dry clutch control based on electromechanical actuator position feedback loop", International journal of vehicle design, vol. 60, no. 3-4, (2012), pp. 305-326.

[22] X. D. Wang, X. P. Xie, X. G. Wu and T. W. Yu, "Precise position tracking control based on adaptive Neuron PID Algorithm for Automatic Clutch Driven by DC Motor", Vehicle Power and Propulsion Conference, (2008).

[23] M. P. Aghababa, "No-chatter variable structure control for fractional nonlinear complex systems", Nonlinear Dynamics, vol. 73, (2013), pp. 2329-2342.

[24] T. L. Chern and Y. C. Wu, "Design of integral variable structure controller and application to electro hydraulic velocity servo systems", IEE Proceedings, (1991).

[25] S. Seshagiri and H. K. Khalil, "Robust output feedback regulation of minimum-phase nonlinear systems using conditional integrators", Automatica, vol. 41, no. 1, (2005), pp. 43-54.

[26] I. Podlubny, "Fractional differential equations", New York: Academic Press, (1999).

[27] B. T. Zhang, Y. G. Pi and Y. Luo, "Fractional order sliding mode control based on parameters autotuning for velocity control of permanent magnet synchronous motor", ISA transactions, vol. 51, (2012), pp. 649-656. 
[28] M. O. Efe, "Fractional fuzzy adaptive sliding mode control of a 2-DOF direct drive robot arm", IEEE Transactions on Systems, Man and Cybernetics, Part B: Cybernetics, vol. 38, no. 6, (2012), pp. 15611570.

[29] H. Delavari, R. Ghaderi, A. Ranjbar and S. Momani, "Fuzzy fractional order sliding mode controller for nonlinear systems", Communications in Nonlinear Science and Numerical Simulation, vol. 15, no. 4, (2010), pp. 963-978.

[30] F. Vasca, L. Iannelli, A. Senatore and G. Reale, "Torque transmissibility assessment for automotive dryclutch engagement", Transactions on mechatronics, vol. 16, no. 3, (2012), pp. 564-573.

[31] M. P. Aghababa, "Comments on "fuzzy fractional order sliding mode controller for nonlinear systems" [commun nonlinear sci numer simulat 15 (2010) 963-978], Commun. Nonlinear Sci. Numer. Simulat, vol. 17, (2012), pp. 1489-1492.

[32] D. Matignon, "Stability results for fractional differential equations with applications to control processing", Computational Engineering in Systems and Application multi-conference, IEEE-SMC Proceedings, (1996); Lille, France.

[33] B. Bonilla, M. Rivero and J.J. Trujillo, "On systems of linear fractional differential equations with constant coefficients", Applied Mathematics and Computation, vol. 187, (2007), pp. 68-78.

[34] E. Ahmed, A.M.A. Sayed and H. A.A. Saka, "Equilibrium points, stability and numerical solutions of fractional-order predator-prey and rabies models", Journal of Mathematical Analysis and Applications, vol. 325, (2007), pp. 542-553.

[35] S. Hosseinnia, H. Ghaderi, R. Ranjbar, A, Ranjbar, F. Abdous and S. Momani, "Control of chaos via fractional-order state feedback controller", New Trends in Nanotechnology and Fractional Calculus Applications. Springer Netherlands, (2010), pp. 511-519.

[36] I. Petras, "Stability of fractional-order systems with rational orders : a survey", Fract. Calc. Appl. Anal. vol. 12, no. 3, (2009), pp. 269-298.

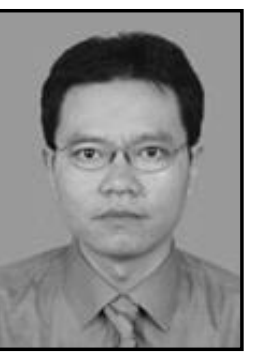

\section{Authors}

Yingchun Long received his Master of Electronics and Communications Engineering (2005) from South China University of Technology. Now he is an associate professor of Physics and Mechanical \& Electrical Engineering, Shao guan University, China. His current research interests include electromechanically systems Control and Artificial Intelligence.

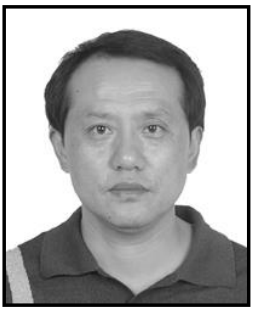

Lifu Li received his Master of Mechanical Engineering and Automation and his Ph.D. of Precise Instruments \& Machinery, both from Chongqing University, in 1991 and 1997, respectively. He is currently a Professor in School of Mechanical and Automotive Engineering South China University of Technology. His current research interests include Optoelectronic \& Mechanical Engineering; industrial computed tomography measurement. 